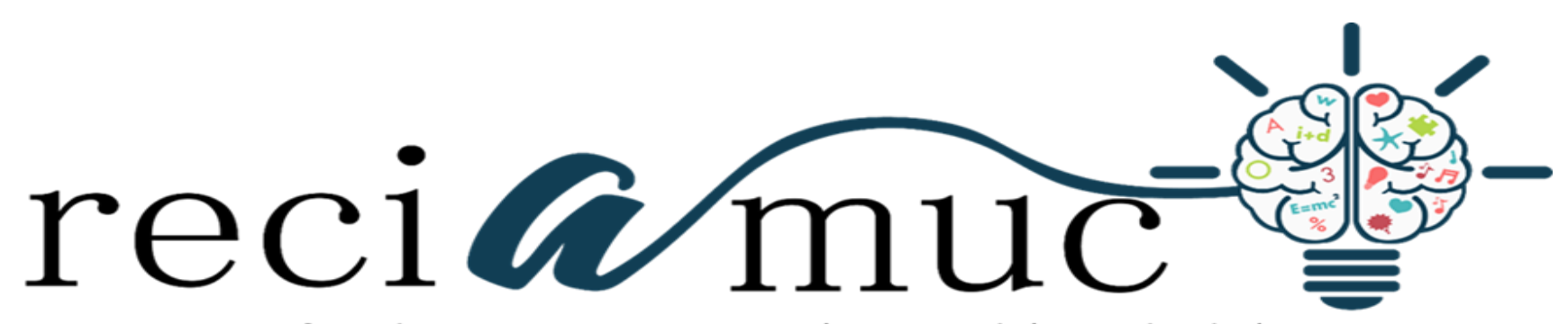

Revista cientifica de investigación actualización del mundo de las ciencias

Valeria Lorena Segarra Zambrano ${ }^{\text {a; }}$ Karen Michelle Bayas Villagomez ${ }^{\text {b; }}$ Adrián

David Gonzaga Ramírez ${ }^{c}$; Sandra Lissette Arregui Romero ${ }^{\text {d }}$

Tratamiento de intoxicación de organofosforado

Organophosphorus poisoning treatment

Revista Científica de Investigación actualización del mundo de las Ciencias. Vol. 3

núm., 2, abril, ISSN: 2588-0748, 2018, pp. 503-530

DOI: $10.26820 /$ reciamuc/3.(2).abril.2019.503-530

URL: $\underline{\text { http://reciamuc.com/index.php/RECIAMUC/article/view/351 }}$

Código UNESCO: 3205 Medicina Interna

Tipo de Investigación: Artículo de Revisión

(ㄱ) RECIAMUC; Editorial Saberes del Conocimiento, 2019

Recibido: 15/02/2019

Aceptado: 07/03/2019

Publicado: 01/04/2019

Correspondencia: valery_1sz@hotmail.com
a. Médico; Investigador Independiente; Guayaquil, Ecuador; valery_1sz@hotmail.com
b. Médico; Investigador Independiente; Guayaquil, Ecuador; karenbayasvillagomez@gmail.com
c. Médico; Investigador Independiente; Guayaquil, Ecuador; adrian_gonzaga@ hotmail.com
d. Médico; Investigador Independiente; Guayaquil, Ecuador; arreguilissette@gmail.com 


\section{Tratamiento de intoxicación de organofosforado}

Vol. 3, núm. 2., (2019)

Valeria Lorena Segarra Zambrano; Karen Michelle Bayas Villagomez; Adrián David Gonzaga Ramírez; Sandra Lissette Arregui Romero

\section{RESUMEN}

Uno de los sectores más importantes para el desarrollo de la economía del país es el agrícola dado que ayuda a satisfacer las necesidades básicas de la sociedad a través de la cosecha de los diversos alimentos. El cultivo de hortalizas, frutas y legumbres tiene un proceso que depende de factores como el estado del clima, temperatura y riego de agua. Otro parámetro importante es el uso de plaguicida que ayudan a controlar la plaga que pueda surgir en los cultivos. El uso de estos plaguicidas debe ser controlado y con la cantidad necesaria para su función principal. Una excesiva aplicación puede acarrear problemas ambientales al ecosistema donde está ubicado el cultivo, así como también intoxicaciones para el ser humano. El plaguicida más importante y de uso más frecuente es el organofosforado por lo que la intoxicación con este plaguicida es más recurrente. El objetivo general de esta investigación es analizar el tratamiento por intoxicación de organofosforado. La metodología empleada se basó en investigaciones de tipo documental y bibliográfica. Los resultados se analizaron bajo las premisas de los tipos de control de plagas, definición del compuesto organofosforado y la intoxicación por este plaguicida. Como conclusión se obtuvo que para poder contrarrestar esta situación se deben aplicar los protocolos que sean necesarios para la atención médica de los pacientes con esta patología. Primeramente, dar los primeros auxilios al momento del ingreso, evaluación física, preguntas sobre síntomas para poder determinar la causa siempre y cuando el paciente este consciente, diagnostico a través de diversos exámenes hematológicos y por último tratamiento que dependerá de la condición del paciente ya que se le puede suministrar atropina y diazepam por si presenta convulsiones.

Palabras clave: Agricultura; Plagas; Plaguicidas; Organofosforado; Tratamiento. 


\title{
Tratamiento de intoxicación de organofosforado
}

Vol. 3, núm. 2., (2019)

Valeria Lorena Segarra Zambrano; Karen Michelle Bayas Villagomez; Adrián David Gonzaga

Ramírez; Sandra Lissette Arregui Romero

\begin{abstract}
One of the most important sectors for the development of the country's economy is agriculture, since it helps meet the basic needs of society through the harvest of various foods. The cultivation of vegetables, fruits and legumes has a process that depends on factors such as weather, temperature and water irrigation. Another important parameter is the use of pesticides that help control the pest that may arise in crops. The use of these pesticides must be controlled and with the amount necessary for their main function. An excessive application can cause environmental problems to the ecosystem where the crop is located, as well as intoxications for the human being. The most important and most frequently used pesticide is the organophosphate, so poisoning with this pesticide is more recurrent. The general objective of this research is to analyze the treatment for organophosphorus poisoning. The methodology used was based on documentary and bibliographic research. The results were analyzed under the premises of the types of pest control, definition of the organophosphorus compound and poisoning by this pesticide. In conclusion, it was obtained that to be able to counteract this situation, the protocols that are necessary for the medical care of patients with this pathology must be applied. First, give first aid at the time of admission, physical evaluation, questions about symptoms to be able to determine the cause as long as the patient is aware, diagnosed through various hematological exams and finally treatment that will depend on the patient's condition since You may be given atropine and diazepam if you have seizures.
\end{abstract}

Key words: Agriculture; Pests; Pesticides; Organophosphate; Treatment. 


\section{Tratamiento de intoxicación de organofosforado}

Vol. 3, núm. 2., (2019)

Valeria Lorena Segarra Zambrano; Karen Michelle Bayas Villagomez; Adrián David Gonzaga Ramírez; Sandra Lissette Arregui Romero

\section{Introducción.}

Una de las fases importantes en el ser humano es la alimentación debido a que es una fuente rica en vitaminas y minerales que permiten dar energía al cuerpo humano para que pueda realizar las diferentes tareas diarias. Una buena alimentación balanceada permite un buen desarrollo de todo el sistema del cuerpo humano y por lo cual genera una buena calidad de vida. Tan esencial es la alimentación en el crecimiento y formación de los individuos en sus primeras etapas de vida.

La producción agrícola juega un papel importante para el desarrollo de la sociedad por lo que sus factores económicos, sociales, y culturales son parte fundamental en el interés de la colectividad. Poder comprender sus fenómenos es parte fundamental de la vida misma. Dentro de los sistemas productivos agrícolas se deben controlar los parámetros que permitan una producción que llene las necesidades de una población. Para ello es necesario poder enfrentar problemas que son comunes en esta área. Un entendimiento profundo de la biología y ecología de los organismos presentes en el agroecosistema resulta en la habilidad de manipularlos y dirigirlos, por lo que el control tradicional simplemente se reacciona, suprimiendo la plaga cuando alcanza altas poblaciones (Jiménez, 2009). Es por ello, que se debe conocer como son las plagas para así comprender y determinar su control y manejo.

Ecuador es un país agrícola donde sus primeras actividades de producción de la economía es la "Agricultura, silvicultura, caza y pesca" por lo que debe generar una alta inversión el uso de plaguicidas para el control de las plagas. La producción de alimentos requiere de una serie de técnicas oportunas para manejar el suelo, agua e insumos químico-orgánicos que conduzcan a lograr buenas cosechas y sin causar daño al ambiente (Basantes Morales, 2015). 


\section{Tratamiento de intoxicación de organofosforado}

Vol. 3, núm. 2., (2019)

Valeria Lorena Segarra Zambrano; Karen Michelle Bayas Villagomez; Adrián David Gonzaga

Ramírez; Sandra Lissette Arregui Romero

Un aspecto importante es el control de plagas dentro de los diversos sistemas agrícolas que tenga la sociedad con el fin de poder obtener los productos alimenticios que necesita la sociedad. Este control debe tener unas características que no perjudiquen los demás aspectos de las comunidades, como la alimentación, la salud y el entorno del ecosistema. Existen métodos de control de plagas y enfermedades más racionales y respetuosas con el medio ambiente y de hecho amigable con la filosofía de desarrollo sustentable y la noción de la conservación de recursos, la biodiversidad y la ética ambiental (Badii \& Abreu, 2006).

Poder contrarrestar los efectos del control de plaga y que no afecta la biodiversidad y ecosistemas que arropa a la sociedad es muy importante. Por lo que, el conocimiento de las plagas es fundamental para obtener un buen control de la misma. Esto debe conducir al uso más eficiente de estos productos, que permita su aplicación sólo cuando sea necesario y en estos casos, aplicar dosis mínimas y mediante las técnicas más efectivas (Jiménez, 2009). Esto permitirá que el producto este en contacto con la plaga en las mínimas condiciones.

Lamentablemente, existe un excedente entre el uso de productos para el control y las plagas, por lo que se han producido efectos colaterales a esta relación. Asimismo, la marcada tendencia hacia la agricultura sustentable también ha impulsado a los investigadores en la búsqueda de insecticidas alternativos por fuera del marco de la síntesis orgánica, explorando diferentes sustancias de origen natural como extractos vegetales, aceites insecticidas e insecticidas inorgánicos (Stadler, Buteler, \& Weaver, 2010).

Para poder efectuar este control de plagas es necesario poder conocer los diferentes procesos agrícolas para la producción de los alimentos, así como también la biodiversidad que está 


\section{Tratamiento de intoxicación de organofosforado}

Vol. 3, núm. 2., (2019)

Valeria Lorena Segarra Zambrano; Karen Michelle Bayas Villagomez; Adrián David Gonzaga Ramírez; Sandra Lissette Arregui Romero

involucrada con este proceso. Existen prácticas agrícolas que tienen el potencial de incrementar o inhibir la biodiversidad funcional, así como también tengan la capacidad de subsidiar la sostenibilidad del agroecosistema al proveer servicios ecológicos como el control biológico, el reciclaje de nutrientes, la conservación de suelo y agua, etc. (Nicholls, 2006). Esto se puede observar en la Figura 1, donde está el efecto de las prácticas agrícolas y el manejo del agroecosistema.

Esto, por supuesto, ocasiona problemas al ecosistema y sociedades que viven y trabajan en la producción agrícola. Por lo cual, es necesario poder determinar políticas que permiten el menos impacto ante el ambiente y el individuo. Estas políticas deben controlar lo siguiente:

“1. Problemas agroecológicos: contaminación y degradación de los suelos, resurgencia y resistencia de plagas, eliminación de enemigos naturales y otros.2. Problemas ambientales: Contaminación de cuerpos de agua y envenenamiento de la fauna, erosión de suelos y contaminación atmosférica.3. Problemas de salud pública: Intoxicaciones agudas, enfermedades resultantes de intoxicaciones crónicas (cáncer, esterilidad, enfermedades hepáticas y renales, etc.), aumento de la malaria, etc.4. Problemas económicos: Uso de divisas para compra de plaguicidas.5. Dependencia tecnológica: El uso de insumos importados del extranjero agudizó la dependencia tecnológica en relación a los países desarrollados, los cuales aseguran un mercado para sus productos agroquímicos”. (Jiménez, 2009, pág. 12) 


\section{Tratamiento de intoxicación de organofosforado}

Vol. 3, núm. 2., (2019)

Valeria Lorena Segarra Zambrano; Karen Michelle Bayas Villagomez; Adrián David Gonzaga

Ramírez; Sandra Lissette Arregui Romero

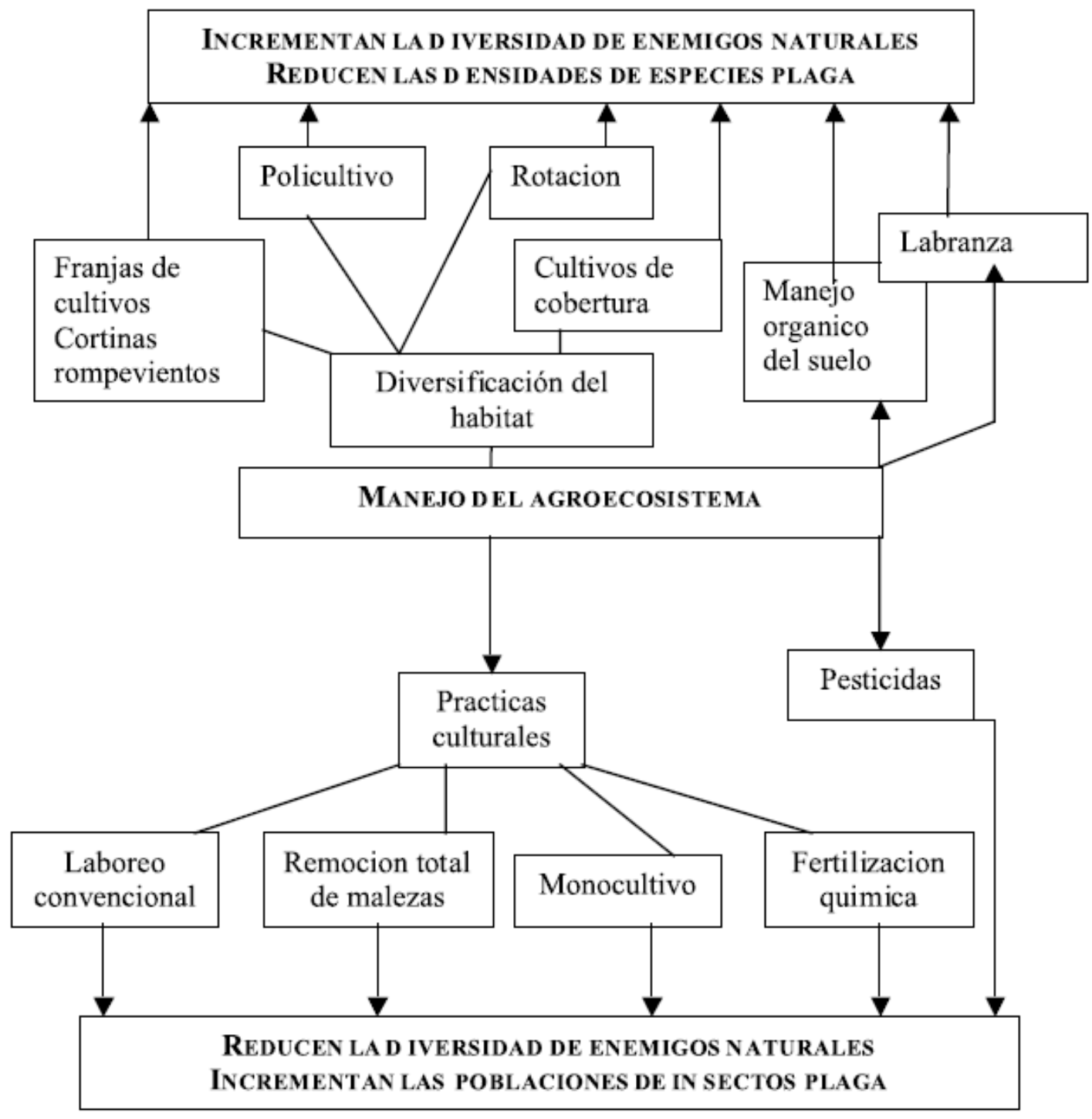

Figura 1. Efecto de las prácticas agrícolas y el manejo del agroecosistema en las poblaciones de insectos plagas y enemigos naturales. Fuente: (Nicholls, 2006)

Las políticas permiten un desarrollo eficiente entre los agricultores, los alimentos y el ecosistema. El control de la plaga debe realizarse en total armonía con los aspectos más sensibles de la sociedad. Como puede apreciarse, existe una política de manejo de plagas en la que se 


\section{Tratamiento de intoxicación de organofosforado}

Vol. 3, núm. 2., (2019)

Valeria Lorena Segarra Zambrano; Karen Michelle Bayas Villagomez; Adrián David Gonzaga Ramírez; Sandra Lissette Arregui Romero

tomanen consideración los aspectos ecológicos, económicos y sociales del control deplagas(Pérez \& Vázquez, 2004).

Para efectuar estas políticas de manejos es necesario comprender a qué tipo de plagas se va a controlar, por lo que es necesario comprender que es este tipo de mal para el cual se debe aplicar mecanismos químicos y físicos para su erradicación. "Plaga es toda aquella población de insectos que ataca a los cultivos establecidos por los seres humanos y cuyo nivel poblacional sube hasta producir una reducción o anulación del rendimiento del cultivo y pérdidas económicas" (Jiménez, 2009).

Existen numerosas plagas que afectan a los cultivos de muchas hortalizas y legumbres, pero se destacan 3 en especial por ser las que atacan a los alimentos más importantes para la sociedad. El gusano cogollero del maíz, Spodoptera frugiperda que atacan a los cultivos del maíz, La mosca blanca, Bemisia tabaco, que afecta a las plantas del tomate, chile, pepino, frijol ytabaco y el picudo del manzano, Amphidees latifrque se come las raíces del árbol (González-Castillo, Aguilar, \& Rodríguez-Herrera, 2012).

Muchos de estas plagas han evolucionado y han permitido una resistencia a los diferentes métodos de erradicación. Según la FAO, es una respuesta disminuida de la población de una especie de animales o plantas a un plaguicida o agente de control como resultado de su aplicación (Bisset, 2002).

Ahora, también existen plagas, que no solo afecta a la agricultura sino también a los urbanismos de las grandes capitales o ciudades. Se presenta una fauna muy variada que puede 


\section{Tratamiento de intoxicación de organofosforado}

Vol. 3, núm. 2., (2019)

Valeria Lorena Segarra Zambrano; Karen Michelle Bayas Villagomez; Adrián David Gonzaga

Ramírez; Sandra Lissette Arregui Romero

producir situaciones de rechazo sin distinguir claramente entre lo que se podría denominar fauna nociva (plagas, fundamentalmente) y fauna beneficiosa (en algunos casos origen también de algunos problemas de salud como las fobias (Moreno Marí, Oltra Moscardó, Falcó Garí, \& Jiménez Peydró, 2007).

Lo importante, es el manejo del control de las plagas por lo que se convierte en un sistema de necesaria transformación cultural. Poder controlar las plagas requiere de compromiso. Un exceso de los métodos de control puede producir contaminación al ecosistema, daño a la salud del agricultor o de la comunidad y puede producir una resistencia de la plaga a la técnica aplicada. Para ello es necesario aplicar medidas curativas y preventivas del control de plagas. Estas se pueden visualizar en la Figura 2.
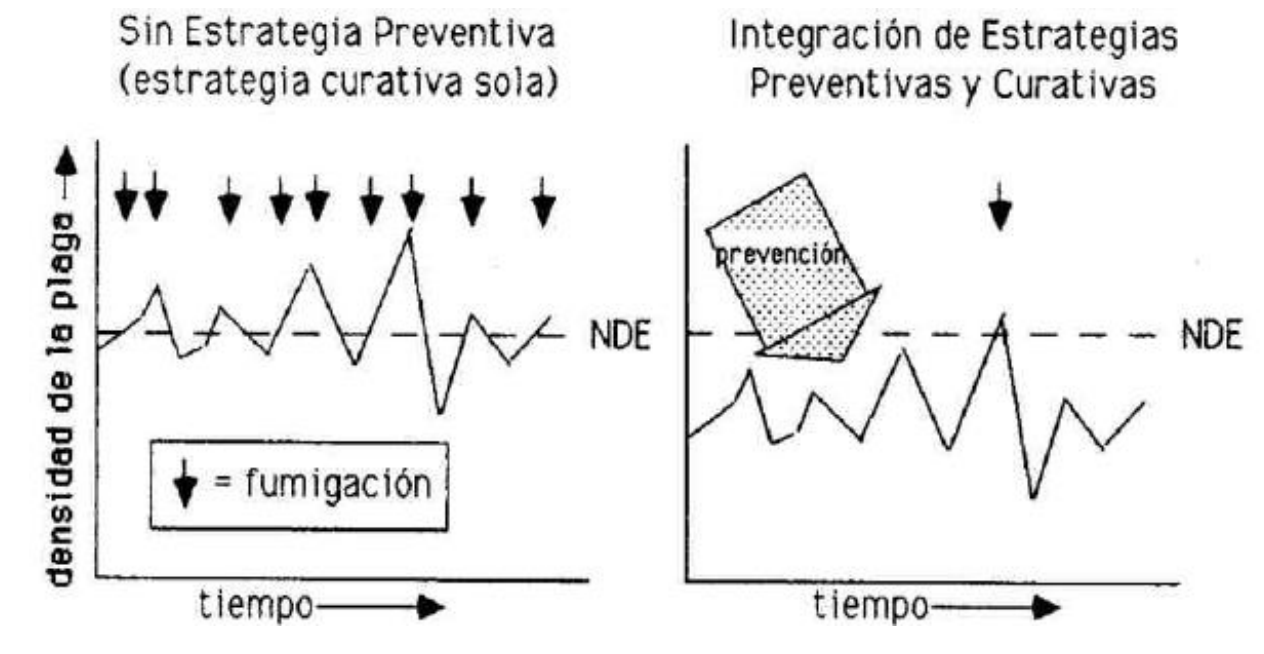

Figura 2. Integración de las estrategias preventivas y curativas en un programa de manejo integrado de plagas. Fuente: (Rosset, 1988)

Indistintamente del lugar donde estén ubicadas las plagas deben aplicarse tácticas de su manejo con el fin de que no se propaguen en todas las áreas. Entre estas tácticas se tienen el control 


\section{Tratamiento de intoxicación de organofosforado}

Vol. 3, núm. 2., (2019)

Valeria Lorena Segarra Zambrano; Karen Michelle Bayas Villagomez; Adrián David Gonzaga Ramírez; Sandra Lissette Arregui Romero

cultural, control mecánico, control físico, control biológico, control genético y control químico (Jiménez, 2009).

Uno de los procesos de control químico es la utilización de plaguicidas. Las intoxicaciones por insecticidas, entre ellos los organofosforados, hacen parte de la lista de eventos de notificación obligatoria a nivel nacional, la cual se pueden clasificar según su toxicidad, su naturaleza química o su función (Fernández, Mancipe, \& Fernández, 2010). Los plaguicidas organofosforados son los más utilizados por su efectividad en el control de las plagas, pero producen efectos en la salud debido a productos contaminados por este plaguicida, así como el contacto durante su manejo y aplicación en los campos agrícolas (Noriega, Armienta, \& Cervantes, 2007).

Por lo tanto, esta investigación tiene como objetivo general analizar el tratamiento por intoxicación de organofosforado. La metodología empleada se basa en investigaciones de tipo documental y bibliográfica.

\section{Método.}

La investigación se derivó de la necesidad de conocer el control de plagas en los cultivos, analizar la importancia y funcionalidad del compuesto organofosforado, determinar la toxicidad del plaguicida y comprender los síntomas diagnósticos y tratamientos para intoxicación de organofosforados. La metodología empleada se basó en investigaciones de tipo documental y bibliográfica tomando en consideración herramientas metodológicas, tales textos, documentos y artículos científicos publicados disponibles en la web. 


\section{Tratamiento de intoxicación de organofosforado}

Vol. 3, núm. 2., (2019)

Valeria Lorena Segarra Zambrano; Karen Michelle Bayas Villagomez; Adrián David Gonzaga

Ramírez; Sandra Lissette Arregui Romero

\section{Resultados.}

Tipos de control de plagas.

Existen muchos tipos de control de plaguicidas donde se destacan principalmente los biológicos. Con el empleo del control biológico se intenta restablecer el perturbado equilibrio ecológico, mediante la utilización de organismos vivos o sus metabolitos, para eliminar o reducir los daños causados por organismos perjudiciales (Badii \& Abreu, 2006).

Indistintamente, para el control de plagas es necesario buscar un equilibrio entre el ambiente, la producción y el individuo. Por tal motivo se debe considerar: Acción específica sobre el objetivo., el impacto bajo o nulo en organismos circundantes y el ambiente y el impacto bajo o nulo en el cultivo (Molina, 2001). En la Tabla 1 se puede mostrar los bioinsecticidas a base de hongos entomopatógenos.

"Su uso permite mantener la productividad del campo sin contaminarlo y sin poner en riesgo la salud de la población que entra en contacto directo o en forma indirecta con estos insumos. Sin embargo, es necesario realizar estudios de impacto ambiental del lugar donde se utilicen, ya que si el agente biológico que se está utilizando no es originario de la región donde se esté aplicando, se corre el riesgo de la introducción de nuevas cepas u organismos que pueden en algunos casos, traer consigo un desplazamiento de las especies que ya están establecidas".(NavaPérez, García-Gutiérrez, Camacho-Báez, \& Vázquez-Montoya, 2012, pág. 26)

También existen controles modernos que permiten una mayor eficiencia en la erradicación de las plagas y por ende una mejor calidad dentro de los factores que pueden perjudicar al 


\section{Tratamiento de intoxicación de organofosforado}

Vol. 3, núm. 2., (2019)

Valeria Lorena Segarra Zambrano; Karen Michelle Bayas Villagomez; Adrián David Gonzaga Ramírez; Sandra Lissette Arregui Romero

ecosistema y a la salud de la sociedad. Estas nuevas técnicas involucran a la nanotecnología. Los nanoinsecticidas, tienen una actividad insecticida (250mg.kg) que es mayor a la obtenida a través de los polvos insecticidas disponibles en el mercado (500 a 5000mg.kg), cuya eficacia depende además de la composición mineral del polvo y del tipo de formulación (Stadler, Buteler, \& Weaver, 2010).

Un factor importante es la producción de los insecticidas. Esto genera que los productos manufacturados con destino a los campos o zonasurbanas tengan la capacidad de afrontar los problemas que puedan ocurrir. Para ello nace la química verde, la cual se basa en la utilización de los productos químicos menos perjudiciales al ambiente y salud del individuo.

“También llamada química sustentable, la química verde es la química aplicada en pro de la prevención de la contaminación. Implica la utilización de una serie de principios encaminados a reducir o eliminar el uso y generación de sustancias peligrosas en el diseño, manufactura y aplicación de los productos químicos, en lugar de recurrir a posteriori al tradicional tratamiento de efluentes; es por ello que la química verde aborda el concepto de prevención en lugar de la remediación”. (Pérez, Ruiz, Schneider, Autino, \& Romanelli, 2013, pág. 86)

Aunado al efecto más ecológico que puedan tener los plaguicidas provenientes de esta química verde y sustentable también deben existir estrategias básicas que permitan cosechas limpias y sin residuos tóxicos como: diseño y preparación del huerto, manejo agronómico, control biológico y capacitación-divulgación (Pérez \& Vázquez, 2004). El diseño y preparación del huerto más el manejo agronómico se puede visualizar en la Tabla 2. 


\section{Tratamiento de intoxicación de organofosforado}

Vol. 3, núm. 2., (2019)

Valeria Lorena Segarra Zambrano; Karen Michelle Bayas Villagomez; Adrián David Gonzaga Ramírez; Sandra Lissette Arregui Romero

Esto permitirá un control de calidad óptimo en la cosecha de los productos agrícolas. Este control basado en la evaluación del rendimiento y en la viabilidad. Se considera de calidad aquel rendimiento que no sea inferior al rendimiento promedio de la cepa y que la viabilidad no sea menor al 95\%(Jiménez, 2009).

Ahora, en muchos países existen leyes y normas implementadas por entes gubernamentales que rigen el uso de los plaguicidas, y aún más pueden autorizar el producto mediante la evaluación exhaustiva de los mismos. El uso de plaguicidas no autorizados podría traer como consecuencia una contaminación injustificada del medio ambiente y una selección de genes de resistencia innecesari (Bisset, 2002). 


\section{Tratamiento de intoxicación de organofosforado}

Vol. 3, núm. 2., (2019)

Valeria Lorena Segarra Zambrano; Karen Michelle Bayas Villagomez; Adrián David Gonzaga Ramírez; Sandra Lissette Arregui Romero

Tabla 1. Bioinsecticidas a base de hongos entomopatógenos 


\section{Tratamiento de intoxicación de organofosforado}

Vol. 3, núm. 2., (2019)

Valeria Lorena Segarra Zambrano; Karen Michelle Bayas Villagomez; Adrián David Gonzaga

Ramírez; Sandra Lissette Arregui Romero

\begin{tabular}{|c|c|c|c|}
\hline Agente biológico & Nombre comercial & Huéspedes & País \\
\hline Beauveria bassiana & BEA-SIN & Lepidópteros & $\begin{array}{l}\text { México- } \\
\text { Sinaloa }\end{array}$ \\
\hline B. bassiana & $\begin{array}{l}\text { AGO } \\
\text { BIOCONTROL }\end{array}$ & $\begin{array}{l}\text { Coleóptera/ } \\
\text { Hemíptera/ } \\
\text { Lepidóptera/ Díptera }\end{array}$ & Colombia \\
\hline B. bassiana & OSTRINIL & Ostrinia nubilalis & Francia \\
\hline B. bassiana & $\begin{array}{l}\text { MYCOTROL WP Y } \\
\text { ES } \\
\text { BOTANICAL } \\
\text { GARD ES, } \\
\text { CORNGARD ES }\end{array}$ & $\begin{array}{l}\text { Hemíptera/ } \\
\text { Heteróptera/ } \\
\text { Coleóptera/ Ortóptera/ } \\
\text { Lepidóptera }\end{array}$ & E.U.A. \\
\hline B. bassiana & BOTANI GARD & $\begin{array}{l}\text { Trips, mosca blanca, } \\
\text { polilla dorso de } \\
\text { diamante }\end{array}$ & Japón \\
\hline B. bongniartii & ENGERLINGSPILZ & $\begin{array}{l}\text { Melolontha } \\
\text { melolontha }\end{array}$ & Suiza \\
\hline B. brongniartii & $\begin{array}{l}\text { AGO- } \\
\text { BIOCONTROL } \\
\text { BEAUVERIA } 50\end{array}$ & $\begin{array}{l}\text { Coleóptera/ } \\
\text { Hemíptera/ Díptera }\end{array}$ & Colombia \\
\hline Beauveria y Metarhizium & $\begin{array}{l}\text { Beauveria Schweizer } \\
\text { Metarhizium } \\
\text { Schweizer }\end{array}$ & Insectos/pastos & Suiza \\
\hline Lagenidium giganteum & LAGINEX & Mosquitos & E.U.A. \\
\hline M. anisopliae & SALTGREEN & $\begin{array}{l}\text { Aneolamia spp, } \\
\text { Prosapia }\end{array}$ & $\begin{array}{l}\text { México- } \\
\text { Córdoba }\end{array}$ \\
\hline M. anisopliae & BIOGREEN & Adoryphouse couloni & Australia \\
\hline M. anisopliae & FITOSAN & Phyllophaga spp. & $\begin{array}{l}\text { México- } \\
\text { Guanajuato }\end{array}$ \\
\hline M. anisopliae & GREEN MUSCLE & $\begin{array}{l}\text { Locusta pardalina y } \\
\text { otras langostas y } \\
\text { chapulines }\end{array}$ & Sudáfrica \\
\hline Isaria fumosorosea & $\begin{array}{l}\text { AGO- } \\
\text { BIOCONTROL } \\
\text { PAECILOMYCES } \\
50\end{array}$ & $\begin{array}{l}\text { Coleóptera/ } \\
\text { Nematodos }\end{array}$ & Colombia \\
\hline P. fumosoroseus & PAE-SIN & Mosquita blanca & $\begin{array}{l}\text { México- } \\
\text { Sinaloa }\end{array}$ \\
\hline P. fumosoroseus & PREFERD & $\begin{array}{l}\text { Mosquita blanca, } \\
\text { áfidos }\end{array}$ & Japón \\
\hline Nomurea rileyi & $\begin{array}{l}\text { AGO- } \\
\text { BIOCONTROL } \\
\text { NOMUREA } 50\end{array}$ & Lepidóptera & Colombia \\
\hline Verticillium lecanii & APHIN & Brevycorine brassicae & México \\
\hline$V$. lecanii & VERTALEC & Áfidos & Suiza \\
\hline V. lecanii & MYCOTAL & Mosquita blanca/ trips & Holanda/Suiza \\
\hline Lecanicillium longisporum & VERTALEC & Afidos & Japón \\
\hline
\end{tabular}

Fuente: (Nava-Pérez, García-Gutiérrez, Camacho-Báez, \& Vázquez-Montoya, 2012) 


\section{Tratamiento de intoxicación de organofosforado}

Vol. 3, núm. 2., (2019)

Valeria Lorena Segarra Zambrano; Karen Michelle Bayas Villagomez; Adrián David Gonzaga Ramírez; Sandra Lissette Arregui Romero

Tabla 2. Prácticas culturales utilizadas en huertos urbanos

\begin{tabular}{|c|c|}
\hline Prácticas & Efectos sobre organismos nocivos \\
\hline Lugar libre de nemátodos & Evita afectaciones por Meloidogyne spp. \\
\hline Terreno nivelado & Reduce desarrollo de enfermedades \\
\hline $\begin{array}{l}\text { Materia orgánica libre de nemátodos y } \\
\text { malezas }\end{array}$ & $\begin{array}{l}\text { Evita afectaciones por Meloidogyne spp. } \\
\text { Limita contaminación con semillas de } \\
\text { malezas }\end{array}$ \\
\hline $\begin{array}{l}\text { Pasillos y alrededores libres de } \\
\text { malezas }\end{array}$ & $\begin{array}{l}\text { Limita acceso de plagas } \\
\text { Reduce fuentes de inóculo de nemátodos y } \\
\text { de enfermedades } \\
\text { Limita acceso de vectores y contamina- } \\
\text { ción de los canteros. }\end{array}$ \\
\hline $\begin{array}{l}\text { Semillas sanas y de buena } \\
\text { germinación }\end{array}$ & Reduce afectaciones por enfermedades \\
\hline Fechas de siembra adecuadas & $\begin{array}{l}\text { Limita incidencia de plagas } \\
\text { Evita infestaciones residuales }\end{array}$ \\
\hline $\begin{array}{l}\text { Considerar posible efecto de colin- } \\
\text { dancia de canteros en cuanto a plagas }\end{array}$ & $\begin{array}{l}\text { Evita contaminaciones por colindancia } \\
\text { negativa }\end{array}$ \\
\hline Optimización de densidad de siembra & Reduce afectaciones por enfermedades \\
\hline Eliminación manual de malezas & Disminuye efecto de las malezas \\
\hline Eliminación de plantas enfermas & Limita diseminación de enfermedades \\
\hline Eliminación de residuos de cosecha & $\begin{array}{l}\text { Disminuye fuentes de inóculo de nemá- } \\
\text { todos, de enfermedades y niveles de } \\
\text { enmalezamiento }\end{array}$ \\
\hline $\begin{array}{l}\text { Inversión del sustrato de los canteros } \\
\text { dos veces en } 15 \text { dias durante junio- } \\
\text { agosto }\end{array}$ & Reduce poblaciones de Meloidogyne spp. \\
\hline $\begin{array}{l}\text { Empleo de plantas repelentes inter- } \\
\text { caladas y en las cercas perimetrales } \\
\text { como caléndula, incienso, albahaca, } \\
\text { orégano, vetiver }\end{array}$ & $\begin{array}{l}\text { Barrera y repelencia de plagas emigrantes } \\
\text { Plantas trampa. }\end{array}$ \\
\hline $\begin{array}{l}\text { Rotación con ajo, cebolla, ajo de } \\
\text { montaña, col, ajonjolí }\end{array}$ & Reduce Meloidogyne spp. \\
\hline $\begin{array}{l}\text { Asociaciones con flor de muerto } \\
\text { (Tagetes) }\end{array}$ & $\begin{array}{l}\text { Reduce Meloidogyne spp. y plagas } \\
\text { insectiles }\end{array}$ \\
\hline Solarización de los canteros & $\begin{array}{l}\text { Baja poblaciones de Meloidogyne spp. y } \\
\text { de malezas }\end{array}$ \\
\hline $\begin{array}{l}\text { Uso de variedades tolerantes o } \\
\text { con resistencia parcial }\end{array}$ & Reduce pérdidas por enfermedades \\
\hline Control del riego (humedad del sustrato) & Limita incidencia de enfermedades \\
\hline
\end{tabular}

Fuente: (Pérez \& Vázquez, 2004)

¿Qué son los plaguicidas organofosforados?

Una de los principales problemas de la producción agrícola es la intoxicación por el uso inconsciente de los plaguicidas, incrementando la tasa de mortalidad a nivel mundial 


\section{Tratamiento de intoxicación de organofosforado}

Vol. 3, núm. 2., (2019)

Valeria Lorena Segarra Zambrano; Karen Michelle Bayas Villagomez; Adrián David Gonzaga

Ramírez; Sandra Lissette Arregui Romero

por lo cual se debe realizar un diagnóstico y tratamiento médico para evitar daños en la salud. En la actualidad los insecticidas organofosforados (IOF) son los plaguicidas empleados con mayor frecuencia en todo el mundo, y por ello, son frecuentes las intoxicaciones por estas sustancias, siendo las responsables de casi el $80 \%$ de las intoxicaciones por plaguicidas que requieren atención médica y del 75\% de las muertes por plaguicidas (Rodríguez Baño, 2013).

“Los compuestos organofosforados son ésteres del ácido fosfórico y de sus derivados, que comparten como característica farmacológica la acción de inhibir enzimas con actividad esterásica, más específicamente de la acetilcolinesterasa en las terminaciones nerviosas, lo que genera una acumulación de acetilcolina y como consecuencia se altera el funcionamiento del impulso nervioso”. (Fernández, Mancipe, \& Fernández, 2010, pág. 85) Estos organofosforados producen efectos perjudiciales y tóxicos al organismo los cuales se describen a continuación:

“1) Inhibición de la enzima acetilcolinesterasa (AchE), produciendo una sobreestimulación colinérgica, que se dominará el "Síndrome Colinérgico". 2) Acción tóxica directa sobre distintos parénquimas, al igual que cualquier otro tóxico. 3) Disfunción de la placa neuromuscular postsináptica, dando lugar al llamado "Síndrome Intermedio". 4) Inhibición de la enzima esterasa neurotóxica (ENT), produciendo una neuropatía retardada (NR)”. (Pérez, Maldonado, \& Ochea, 2009, pág. 144) 


\section{Tratamiento de intoxicación de organofosforado}

Vol. 3, núm. 2., (2019)

Valeria Lorena Segarra Zambrano; Karen Michelle Bayas Villagomez; Adrián David Gonzaga Ramírez; Sandra Lissette Arregui Romero

Es decir, este compuesto organofosforado altera el metabolismo del individuo generando los radicales libres los cuales son moléculas derivadas del oxígeno que tienen un orbital libre en su último nivel principal de energía. Numerosos procesos patológicos que van desde daños celulares hasta la muerte celular se deben al aumento de radicales libres por estrés oxidativo, produciendo que las principales biomoléculas como ácidos nucleicos, proteínas y lípidos sean atacadas por los radicales libres, en especial el último (Noriega, Armienta, \& Cervantes, 2007). Esto se puede visualizar en la Figura 3.

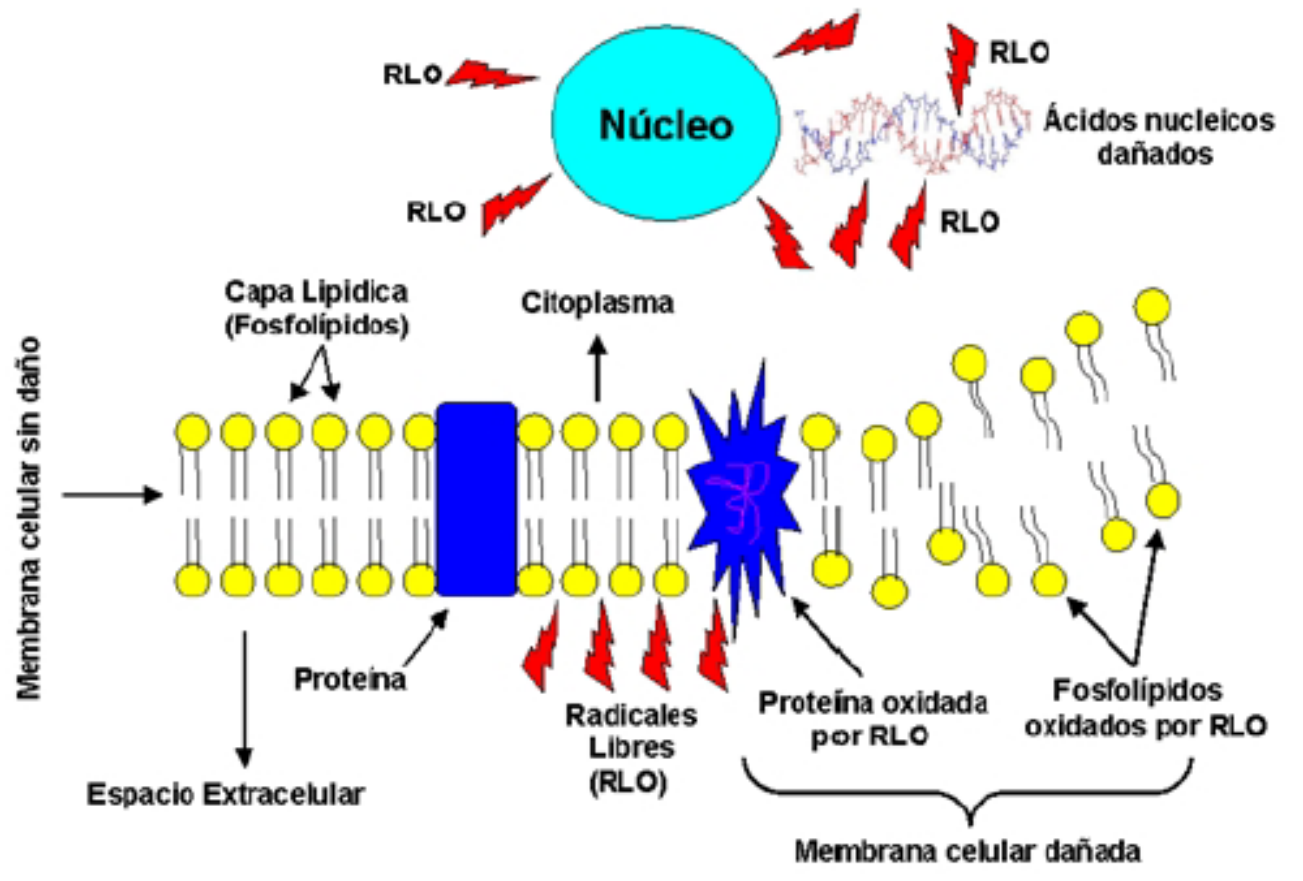

Figura 3. Los radicales libres (RLO) dañan a los ácidos nucleicos y alteran la estructura que conforman a la membrana celular, produciendo un daño directo e irreversible que modifican sus funciones específicas. Fuente: (Noriega, Armienta, \& Cervantes, 2007)

Intoxicación por organofosforado

Los organofosforados presentan una estructura química inestable y se hidrolizan con rapidez por lo que su toxicidad es muy variable, oscilando la dosis potencialmente letal 


\section{Tratamiento de intoxicación de organofosforado}

Vol. 3, núm. 2., (2019)

Valeria Lorena Segarra Zambrano; Karen Michelle Bayas Villagomez; Adrián David Gonzaga

Ramírez; Sandra Lissette Arregui Romero

por vía oral (Andonaire C, 1997). Para conocer la toxicidad de estos plaguicidas se realizó una clasificación que está dividida en 4 grupos, donde la dosis letal 50 (DL50), la cual produce la muerte al $50 \%$ de la población de animales que se les suministro. Esta clasificación se puede observar en la Tabla 3.

Ahora con esta clasificación toxicológica de los plaguicidas, es importante determinar los plaguicidas organofosforados más comunes en el mercado y por el cual es utilizado por los agricultores. En la Tabla 4 se visualiza estos plaguicidas organofosforados según su toxicidad y nombre comercial.

Tabla 3. Clasificación de los palguicidas según categoría toxicologica.

\begin{tabular}{clc}
\hline Categoría & \multicolumn{1}{c}{ Definición } & $\begin{array}{c}\text { Dosis letal 50 (oral } \\
\text { aguda en ratas) }\end{array}$ \\
\hline I & $\begin{array}{l}\text { Extremadamente } \\
\text { tóxicos }\end{array}$ & $0-5 \mathrm{mg} / \mathrm{kg}$ \\
II & Altamente tóxicos & $5-50 \mathrm{mg} / \mathrm{kg}$ \\
III & Medianamente tóxicos & $50-500 \mathrm{mg} / \mathrm{kg}$ \\
IV & Ligeramente tóxicos & Mayor de $500 \mathrm{mg} / \mathrm{kg}$ \\
\hline
\end{tabular}

Modificado de Instituto Nacional de Salud Colombia - Subdirección de Vigilancia y Control. Intoxicación aguda por plaguicidas. Primer semestre de 2007.

Fuente: (Fernández, Mancipe, \& Fernández, 2010) 


\section{Tratamiento de intoxicación de organofosforado}

Vol. 3, núm. 2., (2019)

Valeria Lorena Segarra Zambrano; Karen Michelle Bayas Villagomez; Adrián David Gonzaga Ramírez; Sandra Lissette Arregui Romero

Tabla 4. Plaguicidas organofosforados más comerciales.

\begin{tabular}{cll}
\hline $\begin{array}{c}\text { Categoría } \\
\text { Toxicológica }\end{array}$ & \multicolumn{1}{c}{$\begin{array}{c}\text { Nombre } \\
\text { genérico }\end{array}$} & \multicolumn{1}{c}{$\begin{array}{c}\text { Nombre } \\
\text { comercial }\end{array}$} \\
\hline I & Diclorvos & Diclorvos, Vapona \\
I & Mevinphos & Mevinfos \\
I & Monocrotofos & Monocrotofos 600 SL \\
I & Metilparation & Metilparation, Folidol \\
I & Paration & Parawet, Folidol \\
I & Metamidofos & Tamarón, Monitor \\
II & Coumafos & Asuntol, CoRal \\
II & Diazinon & Basidon \\
II & Fenthion & Lebaycid 500 SC \\
II & Profenofos & Curacron, Tambo \\
III & Clorpirifos & Arriero 2,5, Lorsban \\
III & Malathion & Malathion, Aucuafin \\
\hline
\end{tabular}

Modificado de Thomson PLMS.A. Diccionario Especialidades Agroquímicas. Edición 16. Bogotá: La Entidad, 2006. De: Ministerio de la Protección Social. Guía de Atención Integral en Salud Ocupacional Basada en la Evidencia para Trabajadores Expuestos a Plaguicidas Inhibidores de la Colinesterasa (organofosforados y Carbamatos) GATISO PIC 2007.

Fuente: (Fernández, Mancipe, \& Fernández, 2010)

La fórmula que deriva de este compuesto organofosforado proviene del ácido fosfórico tal cual se puede visualizar en la estructura química del paratión de la Figura 4. Las intoxicaciones agudas más graves se presentan usualmente en ingestiones suicidas o accidentales, donde las dosis tóxicas varían entre los distintos compuestos, para el caso del paratión es de 0.10g (Ferrer, 2003). 


\section{Tratamiento de intoxicación de organofosforado}

Vol. 3, núm. 2., (2019)

Valeria Lorena Segarra Zambrano; Karen Michelle Bayas Villagomez; Adrián David Gonzaga Ramírez; Sandra Lissette Arregui Romero

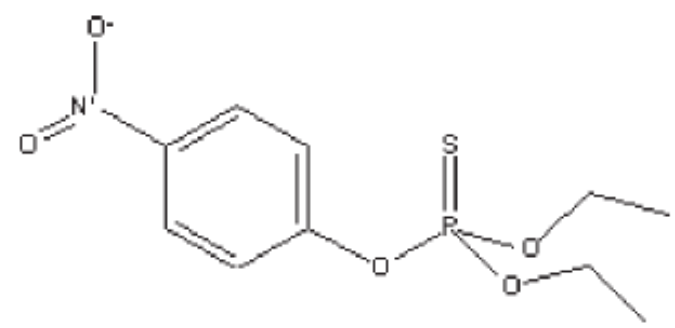

Figura 4. Estructura química del paratión.

Fuente: (Ferrer, 2003)

La patología presente en los individuos que sufren esta enfermedad está relacionada a las manifestaciones de intoxicación la cual dependen de tres síndromes que se superponen. Esto se puede ver en la Tabla 5.

Tabla 5. Manifestaciones de la intoxicación aguda por plaguicidas organofosforado

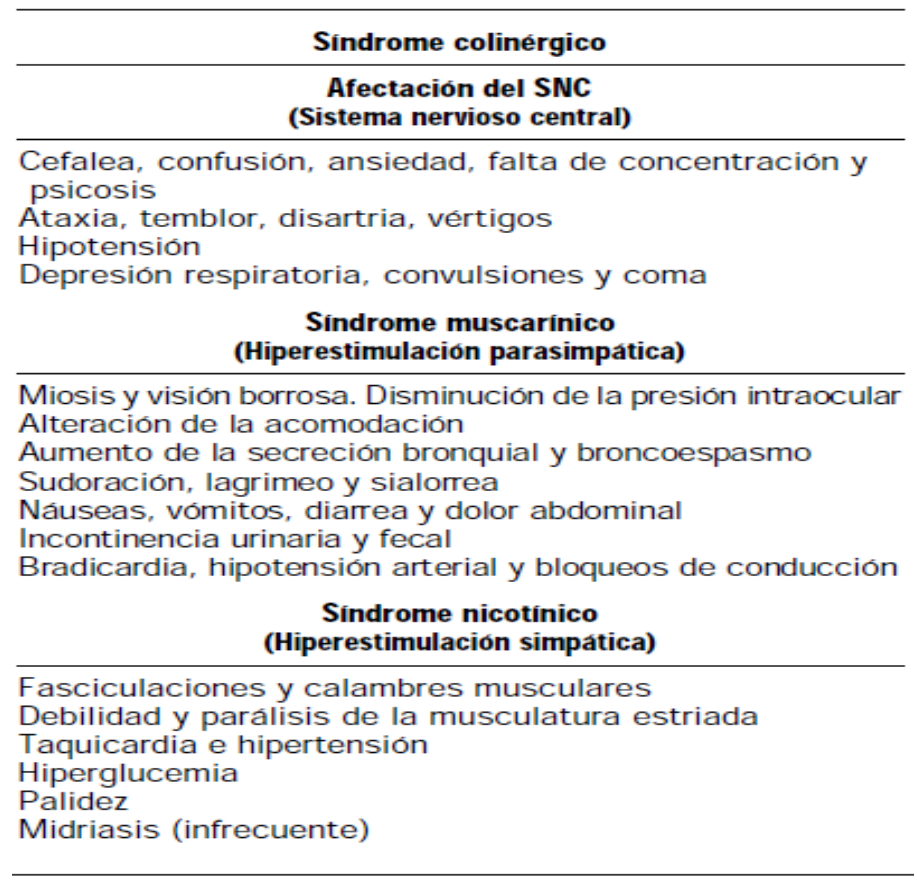

Fuente: (Carod Benedico, 2002) 


\section{Tratamiento de intoxicación de organofosforado}

Vol. 3, núm. 2., (2019)

Valeria Lorena Segarra Zambrano; Karen Michelle Bayas Villagomez; Adrián David Gonzaga Ramírez; Sandra Lissette Arregui Romero

En la Tabla 6, se muestra el diagnostico a pacientes con intoxicación grave de plaguicidas organofosforados.

Tabla 6. Diagnostico por intoxicación grave de plaguicidas organofosforados

\section{DIAGNÓSTICO}

$\begin{gathered}\text { Historia clínica y en relación con exposición } \\ \text { al tóxico. }\end{gathered}$
Manifestaciones clínicas de la intoxicación
aguda por IOP.
Descenso de los niveles séricos de la AC .
Actualmente se investigan distintos marcadores se-
rológicos (esterasa neurotóxica entre otros) de ex-
posición a IOP con objeto de estimar el riesgo de
los trabajadores y establecer un diagnóstico pre-
coz, tienen valor pronóstico.
Mejoría de los síntomas tras administración
de atropina.

Fuente: (Carod Benedico, 2002)

Después de los resultados del diagnóstico se procede a realizar el tratamiento para ayudar a mejorar la calidad de vida y a su vez la eliminación del toxico. Ambos se ven reflejados en la Tabla 7. 


\section{Tratamiento de intoxicación de organofosforado}

Vol. 3, núm. 2., (2019)

Valeria Lorena Segarra Zambrano; Karen Michelle Bayas Villagomez; Adrián David Gonzaga Ramírez; Sandra Lissette Arregui Romero

Tabla 7. Tratamiento y eliminación del tóxico.

\begin{tabular}{|c|}
\hline TRATAMIENTO \\
\hline $\begin{array}{l}\text {-Asegurar la permeabilidad y control de la vía } \\
\text { aérea así como la ventilación del paciente. En caso } \\
\text { de ser precisa intubación, no usar fármacos relajan- } \\
\text { tes musculares. }\end{array}$ \\
\hline $\begin{array}{l}\text { Control precoz de la función cardiovascular } \\
\text { (sobre todo las bradiarritmias). }\end{array}$ \\
\hline $\begin{array}{l}\text {-A nivel extrahospitalario y durante el traslado } \\
\text { está justificado el uso de atropina y la oxigenotera- } \\
\text { pia. Posición más adecuada para el traslado: en de- } \\
\text { cúbito lateral. }\end{array}$ \\
\hline ELIMINACIÓN DEL TÓXICO \\
\hline $\begin{array}{l}\text { 1. Retirar a la persona de la zona contaminada, } \\
\text { principal en vía de entrada respiratoria. } \\
2 \text {. Quitar la ropa y lavar bien la superficie corpo- } \\
\text { ral, secar y tapar. Fundamental en vía de entrada } \\
\text { cutáneo-mucosa. } \\
3 \text {. En caso de ingestión, con paciente consciente, } \\
\text { se puede inducir el vómito en el medio extrahospi- } \\
\text { talario utilizando como único diluyente el agua y si } \\
\text { el traslado a un servicio de urgencias especializado } \\
\text { puede demorarse en exceso. Siempre será de elec- } \\
\text { ción practicar el lavado gástrico con carbón activa- } \\
\text { do en un hospital. } \\
4 \text {. El personal sanitario tomará precauciones } \\
\text { cuando atienda a estos enfermos y solicitará la eti- } \\
\text { queta identificativa del producto. }\end{array}$ \\
\hline
\end{tabular}

Fuente: (Carod Benedico, 2002)

Es importante resaltar que en los servicios de emergencias de los centros de salud se disponga de un plan de atención médica para pacientes con intoxicación por compuesto organofosforado. Esta se muestra en la Tabla 8. 


\section{Tratamiento de intoxicación de organofosforado}

Vol. 3, núm. 2., (2019)

Valeria Lorena Segarra Zambrano; Karen Michelle Bayas Villagomez; Adrián David Gonzaga Ramírez; Sandra Lissette Arregui Romero

Tabla 8. Plan de atención médica para pacientes que sufren de intoxicación por organofosforado

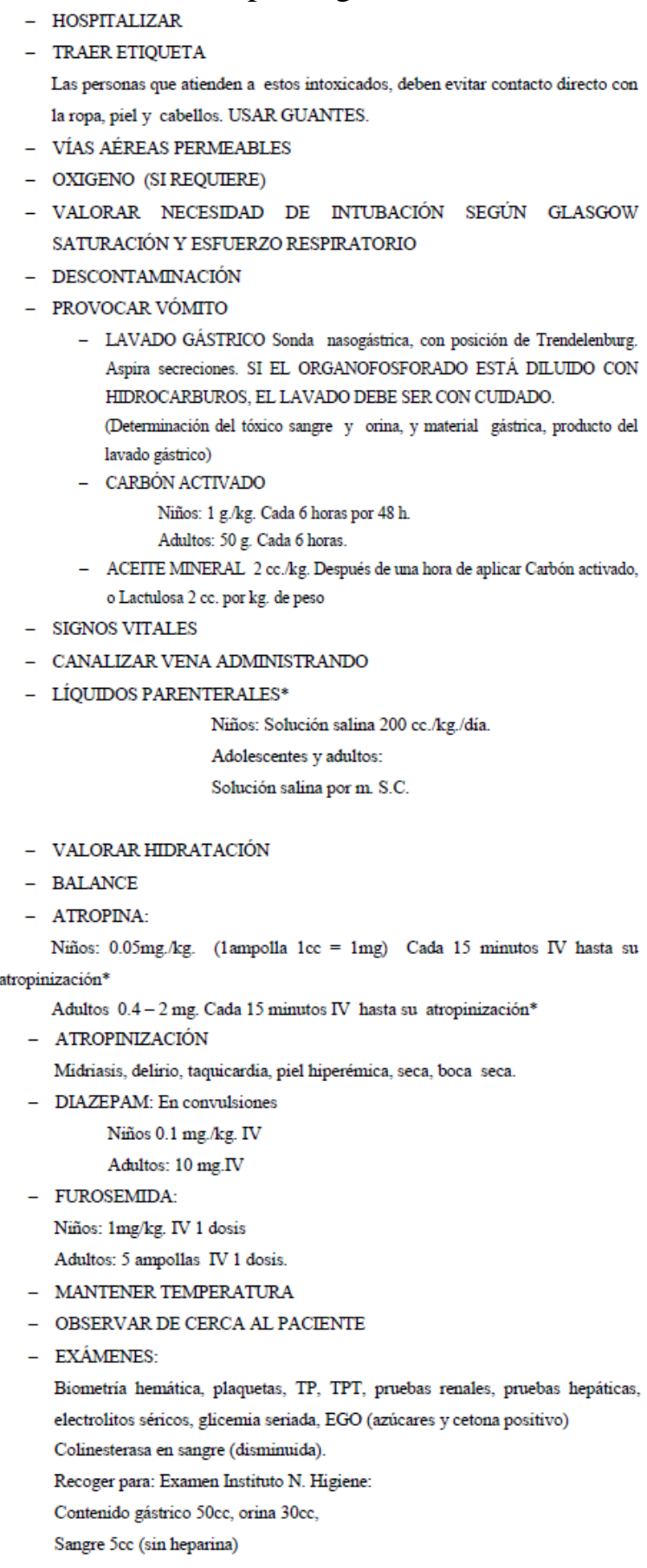

Fuente: (Aveiga Valdivieso, 2012) 


\section{Tratamiento de intoxicación de organofosforado}

Vol. 3, núm. 2., (2019)

Valeria Lorena Segarra Zambrano; Karen Michelle Bayas Villagomez; Adrián David Gonzaga

Ramírez; Sandra Lissette Arregui Romero

\section{Discusión y conclusiones}

Muchos de los países de Latinoamérica tienen como primera base de producción el sistema agrícola y Ecuador no escapa a ello. Este importante sector ayuda a incrementar la economía del mercado y a su vez garantiza las necesidades básicas dela población. Por lo cual, su funcionamiento debe ser uno de los más eficientes del país. sin embargo, eso no escapa a las constantes luchas que tienen los trabajadores de este gremio en contra de muchos parámetros que los afecta. Una de estas variables es la lucha contra las plagas que pueden dañar los cultivos y así producir miles de pérdidas en horas hombre y económicas. Esta lucha se ha llevado a un extremo denominado control de plagas. El control busca minimizar la presencia de estas plagas por efecto de diversas técnicas y métodos que permitirán aplicar su erradicación tanto mecánicas como químicas. Dentro de estas técnicas se encuentra el uso de plaguicidas.

El uso del plaguicida debe ser controlado y analizado por expertos del área ya que su uso excesivo puede perjudicar al ecosistema y a la salud del trabajador que lo manipula. Es necesario poder comprender el nivel de toxicidad de estos plaguicidas para así determinar el uso de esto en los cultivos d ellos alimentos. Existen niveles de toxicidad que se basas en el efecto letal que puede tener sobre un ser viviente. A raíz de este nivel se clasifican en 4 grupos, donde el primero es un nivel extremadamente tóxico con una ingesta de $0-5 \mathrm{gr} / \mathrm{Kg}$ para una dosis letal y el cuarto nivel es de $500 \mathrm{gr} / \mathrm{Kg}$ para una dosis letal.

Dentro de estos plaguicidas se encuentran los organofosforados. Estos son los más utilizados en el mercado y por ende tienen una alta incidencia en la intoxicación por este 


\section{Tratamiento de intoxicación de organofosforado}

Vol. 3, núm. 2., (2019)

Valeria Lorena Segarra Zambrano; Karen Michelle Bayas Villagomez; Adrián David Gonzaga Ramírez; Sandra Lissette Arregui Romero

compuesto. Esto compuesto genera en el organismo que se creen radicales libres que provienen del oxígeno y que atacan a los ácidos nucleicos, proteínas y lípidos. Esto produce un daño irreparable a la condición y calidad de salud del individuo que sufre esta intoxicación.

Para poder contrarrestar esta situación se deben aplicar los protocolos que sean necesarios para la atención médica de los pacientes con esta patología. Primeramente, dar los primeros auxilios al momento del ingreso, evaluación física, preguntas sobre síntomas para poder determinar la causa siempre y cuando el paciente este consciente, diagnostico a través de diversos exámenes hematológicos y por último tratamiento que dependerá de la condición del paciente ya que se le puede suministrar atropina y diazepam por si presenta convulsiones. 


\section{Tratamiento de intoxicación de organofosforado}

Vol. 3, núm. 2., (2019)

Valeria Lorena Segarra Zambrano; Karen Michelle Bayas Villagomez; Adrián David Gonzaga

Ramírez; Sandra Lissette Arregui Romero

\section{Bibliografía.}

Andonaire C, B. M. (1997). Fisiopatología de la intoxicación organofosforados. Revista Med, 18(1), 84-92.

Aveiga Valdivieso, A. A. (2012). Aplicación de un protocolo de manejo de intoxicaciones agudas por plaguicidas. Area de emergencias del Hospital del Niño" Francisco Icaza Bustamante", 2010. Guayaquil, Ecuador: Trabajo de Grado - Universidad de Guayaquil. Facultad de Ciencias Médicas.

Badii, M. H., \& Abreu, J. L. (2006). Control biológico una forma sustentable de control de plagas (Biological control a sustainable way of pest control). Daena: International Journal of Good Conscience, 1(1), 82-89.

Basantes Morales, E. R. (2015). Manejo de cultivos andinos del Ecuador. Sangolquí, Ecuador: Universidad de las Fuerzas Armadas ESPE.

Bisset, J. A. (2002). Uso correcto de insecticidas: control de la resistencia. Revista Cubana de Medicina Tropical, 54(3), 202-219.

Carod Benedico, E. (2002). Insecticidas organofosforados:" De la guerra química al riesgo laboral y doméstico". Medifam, 12(5), 51-62.

Fernández, D. G., Mancipe, L. C., \& Fernández, D. C. (2010). Intoxicación por organofosforados. Revista Med, 18(1), 84-92.

Ferrer, A. (2003). Intoxicación por plaguicidas. In Anales del sistema sanitario de Navarra (Vol. 26). Gobierno de Navarra. Departamento de Salud., 155-171.

González-Castillo, M., Aguilar, C. N., \& Rodríguez-Herrera, R. (2012). Control de insectos-plaga en la agricultura utilizando hongos entomopatógenos: retos y perspectivas. Revista científica de la Universidad Autónoma de Coahuila, 4(8), 42-55.

Jiménez, E. (2009). Métodos de control de plagas. Nicaragua.: Universidad Nacional Agraria, Facultad de Agronomía.

Molina, N. (2001). Uso de extractos botánicos en control de plagas y enfermedades. Manejo Integrado de Plagas. No. 59, 76-77.

Moreno Marí, J., Oltra Moscardó, M. T., Falcó Garí, J. V., \& Jiménez Peydró, R. (2007). El control de plagas en ambientes urbanos: criterios básicos para un diseño racional de los programas de control. Revista española de salud pública, 81(1), 15-24. 


\section{Tratamiento de intoxicación de organofosforado}

Vol. 3, núm. 2., (2019)

Valeria Lorena Segarra Zambrano; Karen Michelle Bayas Villagomez; Adrián David Gonzaga Ramírez; Sandra Lissette Arregui Romero

Nava-Pérez, E., García-Gutiérrez, C., Camacho-Báez, J. R., \& Vázquez-Montoya, E. L. (2012). Bioplaguicidas: una opción para el control biológico de plagas. Ra Ximhai, 8(3), 17-29.

Nicholls, C. (2006). Bases agroecológicas para diseñar e implementar una estrategia de manejo de hábitat para control biológico de plagas. Agroecología, 1, 37-48.

Noriega, O. B., Armienta, A. E., \& Cervantes, P. J. (2007). Evaluación del estrés oxidativo en ratones expuestos crónicamente a un plaguicida organofosforado. Bol Med UAS, 15(2), 811.

Pérez, M. E., Ruiz, D. M., Schneider, M., Autino, J. C., \& Romanelli, G. (2013). La química verde como fuente de nuevos compuestos para el control de plagas agrícolas. Ciencia en Desarrollo, 4(2), 83-91.

Pérez, M. P., M. A., \& Ochea, L. L. (2009). Recambio sanguíneo en una intoxicación por organofosforado (Pyrinex) Reporte de un caso. Revista de Toxicología, 26(2-3), 144-147.

Pérez, N., \& Vázquez, L. L. (2004). Manejo ecológico de plagas. La Habana. Cuba: CEDAR, 296.

Rodríguez Baño, K. A. (2013). Morbimortalidad neonatal, por intoxicación materna con organofosforado, Hospital Sotomayor, de septiembre del 2012 a febrero del 2013 (Bachelor's thesis, Universidad de Guayaquil. Facultad de Ciencias Médicas. Guayaquil, Ecuador: Trabajo de Grado - Universidad de Guayaquil. Facultad de Ciencias Médicas.

Rosset, P. (1988). Aprovechamiento de la ecología y el comportamiento de los insectos mediante las técnicas de control cultural en el manejo integrado de plagas. San José, Costa Rica: Proyecto MIP/CASTIE.

Stadler, T., Buteler, M., \& Weaver, D. K. (2010). Nanoinsecticidas: Nuevas perspectivas para el control de plagas. Revista de la Sociedad Entomológica Argentina, 69(3-4), 149-156.

$$
\text { (9) }(\Theta \odot
$$

\section{RECONOCIMIENTO-NOCOMERCIAL-COMPARTIRIGUAL}

CC BY-NC-SA

ESTA LICENCIA PERMITE A OTROS ENTREMEZCLAR, AJUSTAR Y CONSTRUIR A PARTIR DE SU OBRA CON FINES NO COMERCIALES, SIEMPRE Y CUANDO LE RECONOZCAN LA AUTORÍA Y SUS NUEVAS CREACIONES ESTÉN BAJO UNA LICENCIA CON LOS MISMOS TÉRMINOS. 